\title{
ADP receptor antagonists in patients with acute myocardial infarction complicated by cardiogenic shock: a post hoc IABP-SHOCK II trial subgroup analysis
}

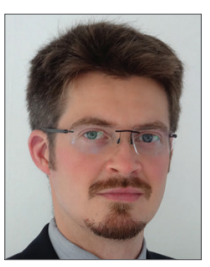

\author{
Martin Orban ${ }^{1 *}, \mathrm{MD}$; Tobias Limbourg${ }^{2}, \mathrm{PhD}$; Franz-Josef Neumann 3 , MD; \\ Miroslaw Ferenc ${ }^{3}$, MD; Hans-Georg Olbrich ${ }^{4}$, MD; Gert Richardt ${ }^{5}$, MD; \\ Marcus Hennersdorf ${ }^{6}$, MD; Klaus Empen 7 , MD; Georg Fuernau ${ }^{8}$, MD; Steffen Desch 9 , MD; \\ Ingo Eitel ${ }^{9}, \mathrm{MD}$; Rainer Hambrecht ${ }^{10}, \mathrm{MD}$; Janine Pöss ${ }^{9}, \mathrm{MD}$; Steffen Schneider ${ }^{2}, \mathrm{PhD}$; \\ Gerhard Schuler ${ }^{8}$, MD; Karl Werdan ${ }^{11}$, MD; Uwe Zeymer ${ }^{2}$, MD; Holger Thiele ${ }^{9}$, MD; \\ Jörg Hausleiter ${ }^{1}, \mathrm{MD}$
}

1. Ludwig-Maximilians-Universität, Munich and German Heart Center Munich, Munich, Germany; 2. Klinikum Ludwigshafen and Institut für Herzinfarktforschung, Ludwigshafen, Germany; 3. Heart Center Bad Krozingen, Bad Krozingen, Germany; 4. Asklepios Clinic Langen-Seligenstadt, Langen, Germany; 5. Heart Center-Segeberger Kliniken, Bad Segeberg, Germany;

6. SLK Kliniken Heilbronn, Heilbronn, Germany; 7. Ernst-Moritz-Arndt University Greifswald, Greifswald, Germany;

8. University of Leipzig - Heart Center, Leipzig, Germany; 9. University Hospital of Schleswig-Holstein (UKSH), Lübeck, Germany; 10. Klinikum Links der Weser, Bremen, Germany; 11. Martin-Luther University Halle-Wittenberg, Halle (Saale), Germany

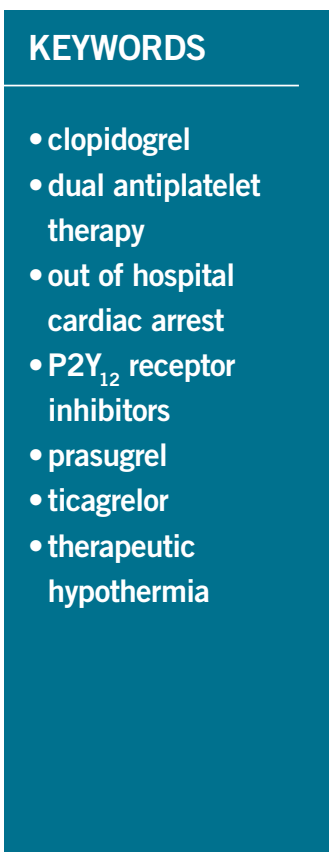

\section{Abstract}

Aims: The aim of this post hoc subgroup analysis of the Intraaortic Balloon Pump in Cardiogenic Shock II trial was to compare the clinical outcome of patients treated with either clopidogrel or the newer, more potent $\mathrm{P} 2 \mathrm{Y}_{12}$ receptor inhibitors prasugrel or ticagrelor.

Methods and results: The primary endpoint was one-year mortality with respect to different $\mathrm{P} 2 \mathrm{Y}_{12}$ receptor inhibitors. Secondary safety endpoints were GUSTO bleedings until hospital discharge. After exclusion of 117 patients (patients who died before or during PCI, patients with unavailable information on $\mathrm{P}_{12}$ receptor inhibitor treatment, patients not receiving or receiving a combination of different $\mathrm{P}_{2} \mathrm{Y}_{12}$ receptor inhibitors as acute antiplatelet therapy), 483 patients were analysed. Of these, 373 patients (77.2\%) received clopidogrel and 110 patients (22.8\%) either prasugrel or ticagrelor as acute antiplatelet therapy. The adjusted rate of mortality did not differ between prasugrel/ticagrelor and clopidogrel treated patients (HR: $0.83,95 \%$ CI: $\left.0.59-1.19, \mathrm{p}_{\mathrm{adj}}=0.31\right)$. GUSTO bleedings did not differ between groups $(14.3 \%$ for prasugrel/ticagrelor and $16.4 \%$ for clopidogrel, HR: $0.91,95 \%$ CI: $\left.0.55-1.5, \mathrm{p}_{\mathrm{adj}}=0.7\right)$.

Conclusions: This IABP-SHOCK II trial subgroup analysis shows that the use of potent $\mathrm{P}_{2} \mathrm{Y}_{12}$ receptor inhibitors like prasugrel or ticagrelor is feasible and might not be harmful in selected patients with cardiogenic shock complicating acute myocardial infarction. However, the superiority in comparison to clopidogrel remains to be proven. ClinicalTrials.gov Identifier: NCT00491036.

*Corresponding author: Medizinische Klinik und Poliklinik I, Klinikum der Universität München, Campus Großhadern, LudwigMaximilians-Universität München, Marchioninistr. 15,81377 Munich, Germany.E-mail: MartinOrban@gmail.com 


\section{Abbreviations}

$\begin{array}{ll}\text { AMI } & \text { acute myocardial infarction } \\ \text { IABP } & \text { intra-aortic balloon pump } \\ \text { PCI } & \text { percutaneous coronary intervention } \\ \text { STEMI } & \text { ST-elevation myocardial infarction }\end{array}$

\section{Introduction}

According to current guidelines, primary percutaneous coronary intervention (PCI) is the recommended reperfusion therapy for patients with acute myocardial infarction (AMI) complicated by cardiogenic shock ${ }^{1}$. Patients undergoing PCI require a dual antiplatelet therapy consisting of acetylsalicylic acid and a $\mathrm{P}_{2} \mathrm{Y}_{12}$ receptor inhibitor ${ }^{2}$. There is a lack of data comparing the safety and efficacy of different $\mathrm{P} 2 \mathrm{Y}_{12}$ receptor inhibitors in the setting of cardiogenic shock. The landmark trials comparing clopidogrel with the potent antiplatelet agents prasugrel (TRial to assess Improvement in Therapeutic Outcomes by optimising platelet inhibitioN with prasugrel-Thrombolysis In Myocardial Infarction, TRITON-TIMI 38) ${ }^{3}$ or ticagrelor (PLATelet inhibition and patient Outcomes, PLATO) ${ }^{4}$ excluded patients with cardiogenic shock complicating AMI. Owing to this circumstance and the limited clinical experience with prasugrel or ticagrelor in shock patients, the majority of cardiogenic shock patients undergoing PCI receive clopidogrel instead of one of the newer antiplatelet agents ${ }^{5}$. In fact, available data on the pharmacodynamic efficacy of clopidogrel in cardiogenic shock patients and in patients after cardiac arrest suggest that the absorption and essential in vivo bioactivation of this prodrug are severely impaired ${ }^{6-11}$.

In this regard the pharmacological properties of the newer, more potent agents are very promising in a shock setting, since prasugrel's metabolisation into the active compound is more rapid and consistent when compared to clopidogrel, and ticagrelor does not require in vivo bioactivation at all. However, despite these advantages over clopidogrel, an impaired absorption of prasugrel and ticagrelor and a reduced in vivo bioactivation of the prodrug prasugrel during cardiogenic shock might delay the onset of their antiplatelet action as well.

Thus, the aim of this post hoc subgroup analysis of the Intraaortic Balloon Pump in Cardiogenic Shock II (IABP-SHOCK II) trial was to compare the clinical outcome of patients with AMI complicated by cardiogenic shock treated with either clopidogrel or the newer, more potent and more rapidly acting $\mathrm{P}_{2} \mathrm{Y}_{12}$ receptor inhibitors prasugrel and ticagrelor.

\section{Methods PATIENTS}

The trial design of the IABP-SHOCK II trial has been published previously ${ }^{12}$. Briefly, it was a prospective, randomised, open-label, multicentre, controlled trial in patients with acute ST-elevation myocardial infarction (STEMI) and non-STEMI complicated by cardiogenic shock planned to undergo early revascularisation (PCI or alternatively bypass surgery; for complete inclusion and exclusion criteria see Thiele et $\mathrm{al}^{12}$ ). All patients were intended to receive optimal medical treatment according to guidelines ${ }^{13}$ plus intensive care treatment according to the German/Austrian S3-Guideline ${ }^{14}$. Six hundred patients with AMI and cardiogenic shock were randomised to IABP or no IABP ${ }^{5}$.

\section{TREATMENT WITH P2Y ${ }_{12}$ RECEPTOR INHIBITORS}

The use of $\mathrm{P} 2 \mathrm{Y}_{12}$ receptor inhibitors was left to the discretion of the attending physicians. There was neither a specific protocol nor randomisation as to which drug to use or when and how to administer.

Since the subgroup of patients treated with the $\mathrm{P}_{2} \mathrm{Y}_{12}$ receptor inhibitor ticagrelor was very small (18 patients, accounting for only $3.7 \%$ of patients), we combined patients receiving prasugrel and ticagrelor treatment resembling the subgroup with the more potent and more rapidly acting $\mathrm{P}_{2} \mathrm{Y}_{12}$ receptor inhibitor treatment (prasugrel/ticagrelor).

\section{STUDY ENDPOINTS, INCLUSION AND EXCLUSION CRITERIA}

The primary endpoint of this post hoc non-randomised observational subgroup analysis was post-procedural one-year mortality with respect to different $\mathrm{P} 2 \mathrm{Y}_{12}$ receptor inhibitors (clopidogrel vs. prasugrel/ticagrelor). Secondary endpoints were 30-day mortality, stroke, myocardial infarction, all bleeding complications and severe or life-threatening bleedings as assessed according to the Global Use of Strategies to Open Occluded Coronary Arteries (GUSTO) criteria ${ }^{15}$ during one-year follow-up in surviving patients. Patients receiving clopidogrel or prasugrel and/or ticagrelor as acute medical therapy qualified for inclusion in the subgroup analysis.

Patients who died before or during PCI were excluded from the primary subgroup analysis as there was no information available regarding the time point of initial $\mathrm{P} 2 \mathrm{Y}_{12}$ receptor inhibitor administration (before or during PCI). Patients with unavailable information on $\mathrm{P}_{2} \mathrm{Y}_{12}$ receptor inhibitor treatment were excluded. Furthermore, we excluded patients not receiving any $\mathrm{P}_{2} \mathrm{Y}_{12}$ receptor inhibitor as acute antiplatelet therapy and patients receiving clopidogrel and prasugrel or ticagrelor simultaneously as acute antiplatelet therapy. Patients receiving prasugrel and ticagrelor simultaneously as acute medical therapy were not excluded from this analysis. Moreover, we investigated the interaction of therapeutic hypothermia on clinical outcome on different $\mathrm{P} 2 \mathrm{Y}_{12}$ receptor inhibitors in this cohort of patients.

A second analysis was performed including all patients with available information on $\mathrm{P}_{2} \mathrm{Y}_{12}$ receptor inhibitor treatment which also included patients who died before and during PCI. Patients receiving clopidogrel and prasugrel or ticagrelor simultaneously as acute antiplatelet therapy were considered as patients treated with prasugrel/ticagrelor. Patients receiving no $\mathrm{P} 2 \mathrm{Y}_{12}$ receptor inhibitor as acute medical therapy were included as a third group (Appendix, Appendix Table 1-Appendix Table 4, Appendix Figure 1-Appendix Figure 4).

Since prasugrel is only recommended in patients $<75$ years of age, we compared baseline characteristics, one-year mortality and bleeding complications of patients treated with clopidogrel and prasugrel $<75$ years of age. Patients treated with ticagrelor were excluded from this second subgroup analysis due to the fact that the use of ticagrelor is not restricted regarding old age. 


\section{STATISTICAL ANALYSIS}

Variables are presented as mean \pm standard deviation (SD), median with interquartile range (IQR) and counts (percentages). Continuously distributed variables were compared using the Mann-Whitney-Wilcoxon test. Categorical variables were compared using the $\chi^{2}$ test, as appropriate. Survival analyses were performed by the Kaplan-Meier method. In addition, a multivariate Cox model with forward selection was used to test for independent predictors of one-year mortality and oneyear bleeding complications. The variables for each model were selected by univariately comparing demographic variables (age, gender), risk factors, medical history, chronic medication and lab parameters from the time of hospital admission between patients with event vs. event-free patients. All variables with a p-value $<0.05$ from this univariate comparison were entered into the Cox regression model (first model), from which the final model was selected by forward selection (with entry level $\mathrm{p}<0.05$ ). For the mortality model, variables entered into the first model were gender, age, mechanical ventilation at admission, history of stroke, history of renal failure, history of coronary artery disease, altered mental status at admission, oliguria, $\mathrm{pH}<7.36$, serum lactate $>2 \mathrm{mmol} / \mathrm{L}$, nicotine as risk factor, arterial hypertension, chronic use of acetylsalicylic acid, beta-blocker, vitamin $\mathrm{K}$ antagonists and lab values at admission from serum creatinine, haemoglobin and haematocrit. A standardised survival curve was produced by means of stratified Cox regression for patients with $\mathrm{pH}$-values $<7.36$, serum lactate $>2 \mathrm{mmol} / \mathrm{L}$ and with medium age (70 years) and medium creatinine values $(114.92 \mu \mathrm{mol} / \mathrm{L})$. Interaction testing was done by entering the interaction term into the respective Cox model. For all statistical analyses, a p-value $<0.05$ was considered significant. All analyses were performed using SAS software SAS, version 9.3 (SAS Institute Inc., Cary, NC, USA).

\section{Results}

\section{STUDY POPULATION}

After exclusion of 117 patients according to exclusion criteria, 483 patients were analysed. Three hundred and seventythree patients $(77.2 \%)$ received clopidogrel and 110 patients $(22.8 \%)$ either prasugrel or ticagrelor as acute antiplatelet therapy (Figure 1).

The baseline characteristics with regard to $\mathrm{P} 2 \mathrm{Y}_{12}$ receptor inhibitor treatment differed significantly between the two groups and are shown in Table 1.

Regarding the clinical presentation prior to randomisation during the primary IABP-SHOCK II trial, patients treated with prasugrel/ticagrelor were more often resuscitated before randomisation, and presented more often with ST-elevation myocardial infarction and with lower levels of creatinine (Table 2). The treatment strategy between the two groups did not differ with respect to PCI, the use of IABP and therapeutic hypothermia. There was a trend towards a more frequent use of glycoprotein (GP) IIb/IIIa inhibitors within the prasugrel/ticagrelor group.
Table 1. Baseline characteristics of the patients.

\begin{tabular}{|c|c|c|c|}
\hline Variable & $\begin{array}{c}\text { Clopidogrel } \\
n=373\end{array}$ & $\begin{array}{c}\text { Prasugrel/ } \\
\text { ticagrelor } \\
n=110\end{array}$ & $p$-value \\
\hline Age, years $\pm S D$ & $68.4 \pm 12.6$ & $62.5 \pm 12.1$ & $<0.0001$ \\
\hline Male gender, $n(\%)$ & $255(68.4)$ & $76(69.1)$ & 0.89 \\
\hline Body mass index*, median [IQR] & $27.7[24.7-30.4]$ & 26.3 [24.2-28.9] & $<0.05$ \\
\hline \multicolumn{4}{|c|}{ Cardiovascular risk factors, n/total n (\%) } \\
\hline Current smoking & 122/371 (32.9) & 47/108 (43.5) & $<0.05$ \\
\hline Hypertension & 264/370 (71.4) & $69 / 110(62.7)$ & 0.08 \\
\hline Hypercholesterolaemia & 137/370 (37.0) & $39 / 110(35.5)$ & 0.76 \\
\hline Diabetes mellitus & 128/371 (34.5) & 21/110 (19.1) & $<0.01$ \\
\hline $\begin{array}{l}\text { Prior myocardial infarction, } \\
\text { n/total n (\%) }\end{array}$ & 87/372 (23.4) & 21/110 (19.1) & 0.34 \\
\hline Prior stroke, n/total n (\%) & $34 / 372(9.1)$ & $0 / 110(0)$ & $<0.01$ \\
\hline $\begin{array}{l}\text { Peripheral arterial disease, } \\
\text { n/total n (\%) }\end{array}$ & 50/372 (13.4) & $10 / 110(9.1)$ & 0.22 \\
\hline Prior PCI, n/total n (\%) & 80/372 (21.5) & $21 / 110(19.1)$ & 0.58 \\
\hline $\begin{array}{l}\text { Prior bypass surgery, } \\
\text { n/total } n(\%)\end{array}$ & 21/372 (5.6) & $2 / 110(1.8)$ & 0.1 \\
\hline Renal impairment, n/total n (\%) & 87/372 (23.4) & $15 / 110(13.6)$ & $<0.05$ \\
\hline \multicolumn{4}{|c|}{ Chronic medical treatment, n/total n (\%) } \\
\hline ASA & $157 / 365(43.0)$ & $34 / 109$ (31.2) & $<0.05$ \\
\hline Beta-blocker & $154 / 365$ (42.2) & $30 / 109$ (27.5) & $<0.01$ \\
\hline ACE inhibitors/ARB & 167/365 (45.8) & 38/109 (34.9) & $<0.05$ \\
\hline Statins & $106 / 365(29.0)$ & $24 / 109(22.0)$ & 0.15 \\
\hline Vitamin $\mathrm{K}$ antagonists & $17 / 365(4.7)$ & 2/109 (1.8) & 0.19 \\
\hline Clopidogrel & $41 / 365$ (11.2) & $7 / 109$ (6.4) & 0.14 \\
\hline Prasugrel & $1 / 365(0.3)$ & 4/109 (3.7) & $<0.01$ \\
\hline Ticagrelor & $1 / 254(0.4)$ & $0 / 86(0)$ & 0.56 \\
\hline
\end{tabular}

Baseline characteristics of the clopidogrel and the prasugrel/ticagrelor subgroups. Data presented are means ( \pm standard deviation $[S D]$ ), medians $[I Q R]$ or numbers of patients (percentages). *The body mass index is the weight in kilograms divided by the square of the height in metres. ACE: angiotensin-converting enzyme; ARB: angiotensin receptor blockers; ASA: acetylsalicylic acid; IQR: interquartile range; PCI: percutaneous coronary intervention

\section{CLINICAL OUTCOME}

Unadjusted all-cause one-year mortality was lower in prasugrel/ ticagrelor vs. clopidogrel treated patients (36.4\% [40/110] vs. $52.5 \%$ [196/373] of patients, $\mathrm{p}<0.01$ ) (survival curve, Figure 2). There was also a lower mortality in prasugrel/ticagrelor vs. clopidogrel treated patients at 30-day follow-up (29.1\% [32/110] vs. $39.4 \%$ [147/373], $<<0.05)$. In a Cox regression analysis with mortality during one year as the dependent variable, the adjusted rate of mortality did not differ between prasugrel/ticagrelor vs. clopidogrel treated patients (HR: 0.83, 95\% CI: 0.59-1.19, $\mathrm{p}=0.31$ ) (standardised survival curve, Figure 3 ). In this analysis, age, a $\mathrm{pH}<7.36$, serum lactate $>2 \mathrm{mmol} / \mathrm{l}$ and serum-creatinine were independent predictors of one-year mortality (Table 3). In a Cox regression analysis with the combined ischaemic secondary endpoint death or MI or stroke during one year as the dependent variable, the adjusted rate of mortality did not differ between 
Table 2. Clinical presentation of both subgroups prior to randomisation during the primary IABP-SHOCK II trial.

\begin{tabular}{|c|c|c|c|}
\hline Variable & $\begin{array}{c}\text { Clopidogrel } \\
\qquad=373\end{array}$ & $\begin{array}{c}\text { Prasugrel/ } \\
\text { ticagrelor } \\
n=110\end{array}$ & $p$-value \\
\hline \multicolumn{4}{|c|}{ Sign of impaired organ perfusion, n/total n (\%) } \\
\hline Altered mental status & $282 / 373(75.6)$ & 79/110 (71.8) & 0.42 \\
\hline $\begin{array}{l}\text { Cold, clammy skin and } \\
\text { extremities }\end{array}$ & $328 / 373(87.9)$ & $87 / 110(79.1)$ & $<0.05$ \\
\hline Oliguria $(\leq 20 \mathrm{ml} / \mathrm{hr})$ & $115 / 373(30.8)$ & $31 / 110$ (28.2) & 0.59 \\
\hline Serum lactate $>2.0 \mathrm{mmol} /$ litre & $292 / 373(78.3)$ & $70 / 110(63.6)$ & $<0.01$ \\
\hline Fibrinolysis*, n/total n (\%) & $35 / 373(9.4)$ & $7 / 110(6.4)$ & 0.32 \\
\hline Resuscitation*, n/total n (\%) & $152 / 373(40.8)$ & $58 / 110(52.7)$ & $<0.05$ \\
\hline $\begin{array}{l}\text { Mechanical ventilation, } \\
\mathrm{n} / \mathrm{total} \mathrm{n}(\%)\end{array}$ & $212 / 373(56.8)$ & $58 / 110(52.7)$ & 0.45 \\
\hline \multicolumn{4}{|l|}{ Myocardial infarction, n/total n (\%) } \\
\hline ST-segment elevation & $218 / 372(58.6)$ & $92 / 109(84.4)$ & $<0.0001$ \\
\hline Anterior & $150 / 371(40.4)$ & $54 / 109(49.5)$ & 0.09 \\
\hline Creatinine $(\mu \mathrm{mol} / \mathrm{L})$, median $[\mathrm{IQR}]$ & 117 [96-153] & $105[86-133]$ & $<0.01$ \\
\hline \multicolumn{4}{|l|}{ №. of diseased vessels, n/total n (\%) } \\
\hline 1 & $78 / 370(21.1)$ & $27 / 110(24.5)$ & 0.44 \\
\hline 2 & $94 / 370(25.4)$ & $33 / 110(30.0)$ & 0.34 \\
\hline 3 & $198 / 370(53.5)$ & $50 / 110(45.5)$ & 0.14 \\
\hline \multicolumn{4}{|l|}{ Treatment strategy, n/total n (\%) } \\
\hline Primary PCl & $363 / 373(97.3)$ & $108 / 110(98.2)$ & 0.61 \\
\hline Primary bypass surgery & $2 / 373(0.5)$ & $2 / 110(1.8)$ & 0.19 \\
\hline Primary conservative & $8 / 373(2.1)$ & $0 / 110(0)$ & 0.12 \\
\hline IABP, n/total n (\%) & 206/373 (55.2) & $55 / 110(50.0)$ & 0.33 \\
\hline $\begin{array}{l}\text { Mild therapeutic hypothermia, } \\
\text { n/total } n(\%)\end{array}$ & $120 / 373(32.2)$ & $38 / 110(34.5)$ & 0.64 \\
\hline $\begin{array}{l}\text { Glycoprotein Ilb/llla inhibitors, } \\
\text { n/total n (\%) }\end{array}$ & $172 / 373(46.1)$ & $62 / 110(56.4)$ & 0.06 \\
\hline \multicolumn{4}{|c|}{$\begin{array}{l}\text { Clinical presentation and acute treatment of both subgroups. *Fibrinolysis }<24 \text { hours before } \\
\text { randomisation and resuscitation before randomisation within the primary IABP-SHOCK II } \\
\text { trial. Data presented are means ( } \pm \text { standard deviation [SD]), medians [IQR] or numbers of } \\
\text { patients (percentages). IABP: intra-aortic balloon pump; IQR: interquartile range; } \\
\text { PCl: percutaneous coronary intervention }\end{array}$} \\
\hline
\end{tabular}

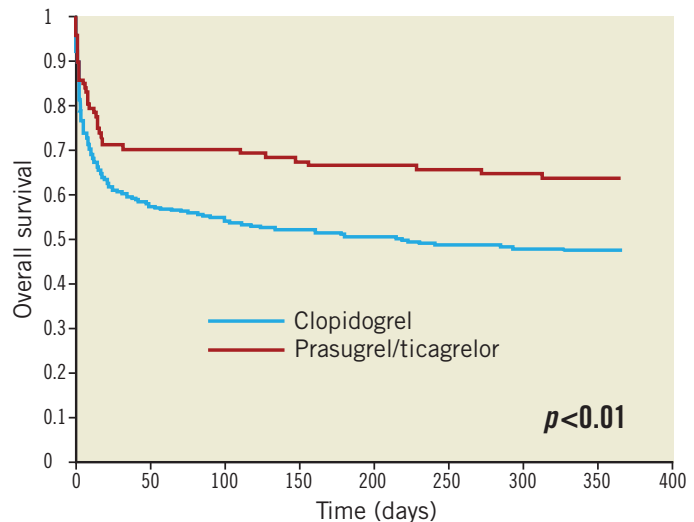

Patients at risk

$\begin{array}{lrrrrrrrr}\text { Clopidogrel } & 373 & 213 & 201 & 192 & 187 & 181 & 178 & 172\end{array}$

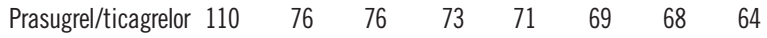

Figure 2. Unadjusted one-year survival. Unadjusted survival curve during one-year follow-up in patients treated with prasugrel or ticagrelor (red line) versus clopidogrel (blue line).

prasugrel/ticagrelor vs. clopidogrel treated patients (HR: 0.85, 95\% CI: 0.61-1.19, $\mathrm{p}=0.33$ ).

The incidence of myocardial infarction and ischaemic stroke did not differ between the subgroups (Table 4).

GUSTO bleedings of surviving patients did not differ between the two subgroups (all GUSTO bleedings: 14.3\% [10/70] of prasugrel/ticagrelor vs. $16.4 \%$ [29/177] of clopidogrel treated patients, $\mathrm{p}=0.68$ ) (Figure 4). In a Cox regression analysis with all GUSTO bleedings during one year as the dependent variable, the adjusted rate of all bleedings did not differ between prasugrel/ticagrelor vs. clopidogrel treated patients (HR: 0.91, 95\% CI: $0.55-1.5, \mathrm{p}=0.70$ ). There was also no difference in GUSTO major bleedings between prasugrel/ticagrelor vs. clopidogrel treated patients $(4.3 \%[3 / 70]$ vs. $2.3 \%[4 / 177], \mathrm{p}=0.39$, respectively) (Figure 5). The rate of mortality, and ischaemic and bleeding

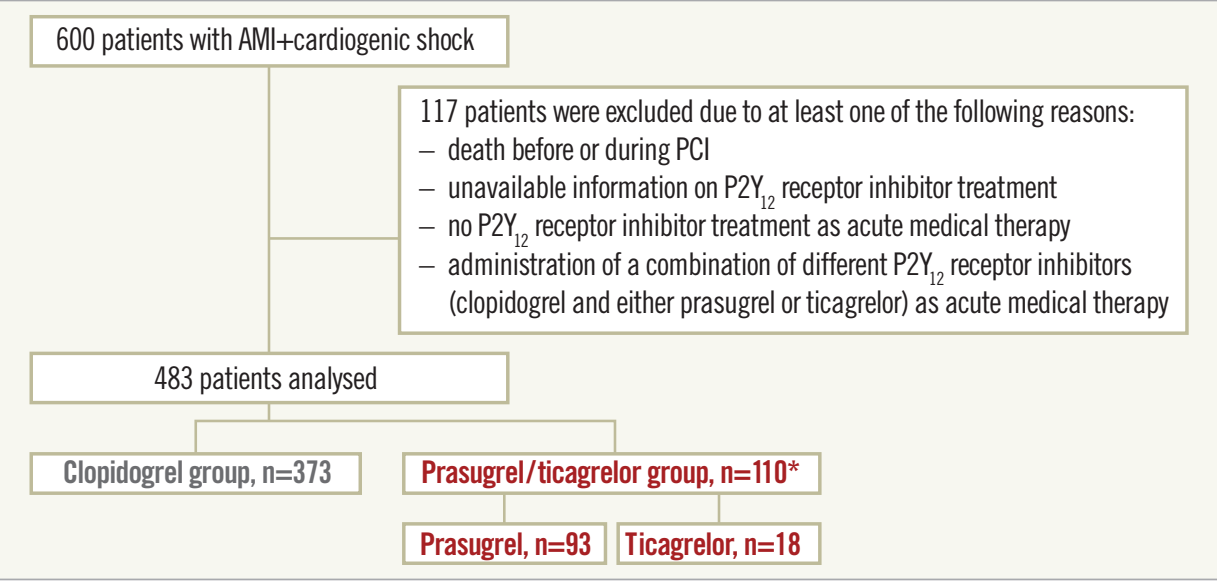

Figure 1. Study flow chart. Study flow chart of patients in the IABP-SHOCK II trial subgroup analysis on P2Y ${ }_{12}$ receptor inhibitors. *One patient received prasugrel and ticagrelor simultaneously as acute medical therapy. AMI: acute myocardial infarction; PCI: percutaneous coronary intervention 
Table 3. Univariate and multivariate Cox model of 12-month mortality.

\begin{tabular}{|c|c|c|c|c|c|}
\hline \multicolumn{2}{|c|}{ Variable } & $\begin{array}{l}\text { Univariate } \\
\text { OR (95\% CI) }\end{array}$ & $p$-value & $\begin{array}{l}\text { Multivariate } \\
\text { HR ( } 95 \% \text { CI) }\end{array}$ & $p$-value \\
\hline \multicolumn{2}{|c|}{ Age (per 10 years) } & $1.45(1.24-1.68)$ & $<0.0001$ & $1.28(1.14-1.44)$ & $<0.0001$ \\
\hline \multicolumn{2}{|l|}{ Female gender } & $1.31(0.89-1.93)$ & 0.17 & & \\
\hline \multirow{4}{*}{$\begin{array}{l}\text { Cardiovascular } \\
\text { risk factors }\end{array}$} & Current smoking & $0.60(0.41-0.88)$ & $<0.01$ & NA & NA \\
\hline & Hypertension & $1.63(1.10-2.42)$ & $<0.05$ & NA & NA \\
\hline & Hypercholesterolaemia & $1.10(0.76-1.59)$ & 0.63 & & \\
\hline & Diabetes mellitus & $1.19(0.81-1.76)$ & 0.37 & & \\
\hline \multicolumn{2}{|c|}{ Prior myocardial infarction } & $1.29(0.84-1.98)$ & 0.24 & & \\
\hline \multicolumn{2}{|l|}{ Prior $\mathrm{PCl}$} & $1.48(0.95-2.30)$ & 0.08 & & \\
\hline \multicolumn{2}{|c|}{ Prior bypass surgery } & $0.80(0.34-1.86)$ & 0.6 & & \\
\hline \multicolumn{2}{|l|}{ Prior stroke } & $2.70(1.26-5.77)$ & $<0.01$ & NA & NA \\
\hline \multicolumn{2}{|c|}{ Peripheral arterial disease } & $2.54(1.42-4.51)$ & $<0.01$ & NA & NA \\
\hline \multicolumn{2}{|l|}{ Renal impairment } & $2.29(1.45-3.60)$ & $<0.001$ & NA & NA \\
\hline \multirow{8}{*}{$\begin{array}{l}\text { Chronic medical } \\
\text { treatment }\end{array}$} & ASA & $1.87(1.29-2.71)$ & $<0.001$ & NA & NA \\
\hline & Beta-blocker & $1.6(1.1-2.32)$ & $<0.05$ & NA & NA \\
\hline & ACE inhibitors/ARB & $1.27(0.88-1.83)$ & 0.2 & & \\
\hline & Statins & $1.42(0.94-2.12)$ & 0.09 & & \\
\hline & Vitamin $\mathrm{K}$ antagonists & $3.13(1.11-8.82)$ & $<0.05$ & NA & NA \\
\hline & Clopidogrel & $1.57(0.86-2.87)$ & 0.14 & & \\
\hline & Prasugrel & $0.26(0.03-2.38)$ & 0.2 & & \\
\hline & Ticagrelor & NA & 0.31 & & \\
\hline \multirow{5}{*}{$\begin{array}{l}\text { Sign of impaired } \\
\text { organ perfusion }\end{array}$} & Altered mental status & $1.66(1.09-2.52)$ & $<0.05$ & NA & NA \\
\hline & Cold, clammy skin and extremities & $1.42(0.85-2.39)$ & 0.18 & & \\
\hline & Oliguria ( $\leq 20 \mathrm{ml} / \mathrm{hr}$ ) & $1.6(1.08-2.37)$ & $<0.05$ & NA & NA \\
\hline & Serum lactate $>2.0 \mathrm{mmol} /$ litre & $2.74(1.77-4.25)$ & $<0.0001$ & $1.84(1.26-2.67)$ & $<0.01$ \\
\hline & $\mathrm{pH}<7.36$ & $1.60(1.10-2.33)$ & $<0.05$ & $1.49(1.1-2.0)$ & $<0.01$ \\
\hline \multicolumn{2}{|l|}{ Fibrinolysis } & $1.31(0.69-2.47)$ & 0.41 & & \\
\hline \multicolumn{2}{|l|}{ Resuscitation } & $1.18(0.82-1.69)$ & 0.38 & & \\
\hline \multicolumn{2}{|c|}{ Mechanical ventilation } & $1.48(1.03-2.13)$ & $<0.05$ & NA & NA \\
\hline \multirow{2}{*}{$\begin{array}{l}\text { Myocardial } \\
\text { infarction }\end{array}$} & ST-segment elevation & $0.59(0.40-0.86)$ & $<0.01$ & NA & NA \\
\hline & Anterior & $0.83(0.58-1.19)$ & 0.31 & & \\
\hline \multicolumn{2}{|c|}{ Creatinine (per $\mu \mathrm{mol} / \mathrm{l})$} & $1.011(1.007-1.015)$ & $<0.0001$ & $1.003(1.002-1.004)$ & $<0.0001$ \\
\hline \multicolumn{2}{|c|}{ Haemoglobin (per mmol/l) } & $0.806(0.711-0.913)$ & $<0.0001$ & NA & NA \\
\hline \multicolumn{2}{|c|}{ Haematocrit (per 10\%) } & $0.792(0.570-0.932)$ & $<0.05$ & NA & NA \\
\hline \multicolumn{2}{|c|}{$\mathrm{P}^{2} \mathrm{Y}_{12}$ treatment (prasugrel/ticagrelor) } & $0.52(0.34-0.81)$ & $<0.01$ & $0.83(0.59-1.19)$ & 0.31 \\
\hline \multicolumn{2}{|l|}{ IABP } & $1.14(0.80-1.64)$ & 0.46 & & \\
\hline \multicolumn{2}{|c|}{ Mild therapeutic hypothermia } & $1.84(1.25-2.71)$ & $<0.01$ & NA & NA \\
\hline \multicolumn{2}{|c|}{ Glycoprotein IIb/IIla inhibitors } & $0.75(0.52-1.07)$ & 0.11 & & \\
\hline
\end{tabular}

events of patients treated with clopidogrel vs. patients treated with prasugrel/ticagrelor are listed in Table 4.

No significant interaction was observed between the use of mild therapeutic hypothermia and the type of thienopyridine (prasugrel/ ticagrelor vs. clopidogrel) with respect to mortality risk $\left(\mathrm{p}_{\mathrm{int}}=0.06\right)$ in this cohort of patients.

\section{CLINICAL OUTCOME OF ALL PATIENTS WITH AVAILABLE INFORMATION ON P2Y ${ }_{12}$ RECEPTOR INHIBITOR TREATMENT}

In a second analysis which included all patients with available information on $\mathrm{P}_{2} \mathrm{Y}_{12}$ receptor inhibitor treatment (598 patients, also including patients who died before and during PCI) receiving either clopidogrel (387 patients) or prasugrel/ticagrelor (125 patients) or 


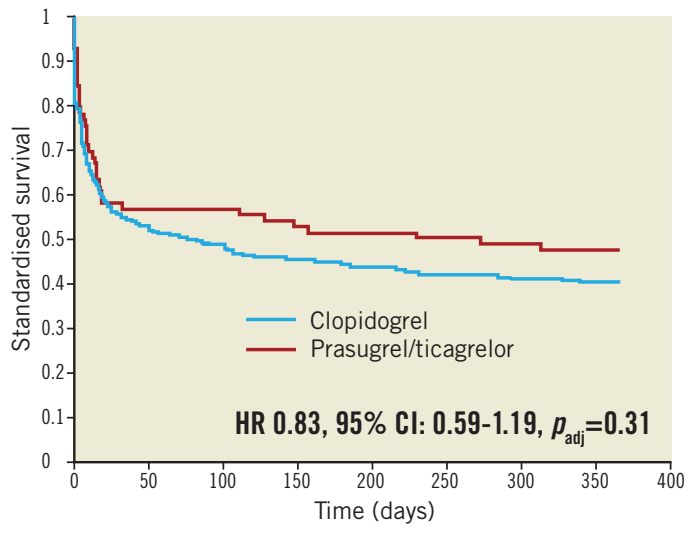

Patients at risk

$\begin{array}{lllllllll}\text { Clopidogrel } & 373 & 213 & 201 & 192 & 187 & 181 & 178 & 172\end{array}$

$\begin{array}{llllllll}\text { Prasugrel/ticagrelor } 110 & 76 & 76 & 73 & 71 & 69 & 68 & 64\end{array}$

Figure 3. Standardised one-year survival. Standardised survival curve (stratified Cox regression for patients with $\mathrm{pH}$-values $<7.36$, serum lactate $>2 \mathrm{mmol} / \mathrm{L}$ and with medium age and medium creatinine values, all measures taken at admission) during one-year follow-up in patients treated with prasugrel or ticagrelor (red line) versus clopidogrel (blue line). CI: confidence interval; HR: hazard ratio

no $\mathrm{P} 2 \mathrm{Y}_{12}$ receptor inhibitor treatment (86 patients), results were consistent compared to the primary analysis (Appendix).

\section{CLINICAL OUTCOME OF PATIENTS < 75 YEARS OF AGE TREATED WITH CLOPIDOGREL VS. PRASUGREL}

Patients treated with prasugrel had a numerically lower - albeit not statistically significant - rate of mortality (32.9\% vs. $44.7 \%$, $\mathrm{p}=0.06)$, but a higher incidence of stroke $(5.3 \%$ vs. $0.7 \%, \mathrm{p}<0.05)$. All other ischaemic and bleeding endpoints were not different between the two groups. In a Cox regression analysis the adjusted

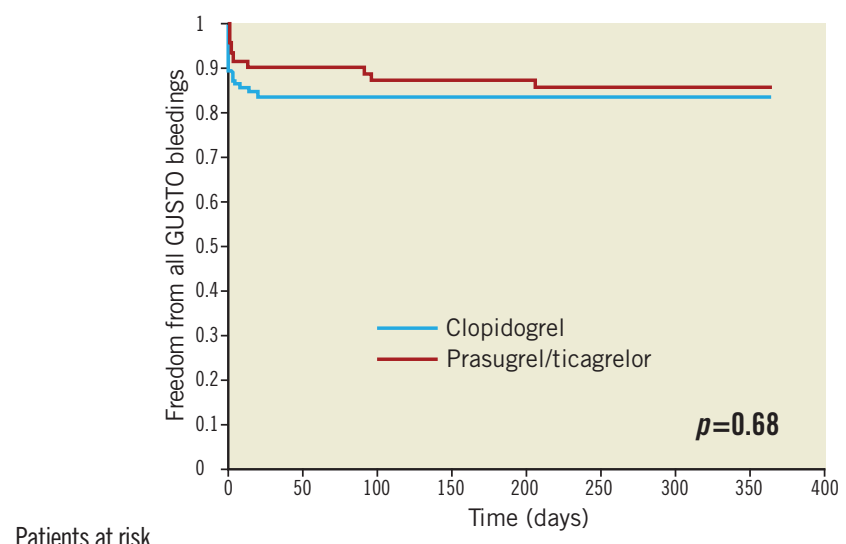

Patients at risk

$\begin{array}{lllllllll}\text { Clopidogrel } & 178 & 173 & 172 & 172 & 172 & 171 & 170 & 166\end{array}$ $\begin{array}{lllllllll}\text { Prasugrel/ticagrelor } & 70 & 68 & 66 & 66 & 65 & 63 & 63 & 61\end{array}$

Figure 4. One-year freedom from all GUSTO bleedings. KaplanMeier curves of freedom from all GUSTO bleedings during one-year follow-up in surviving patients treated with prasugrel or ticagrelor (red line) versus clopidogrel (blue line).
Table 4. Clinical outcome at one year.

\begin{tabular}{|c|c|c|c|}
\hline $\begin{array}{l}\text { Events, n (\%) } \\
12 \text { months }\end{array}$ & $\begin{array}{c}\text { Clopidogrel } \\
n=373\end{array}$ & $\begin{array}{l}\text { Prasugrel } \\
\text { ticagrelor } \\
n=110\end{array}$ & $p$-value \\
\hline Death & $196 / 373(52.5)$ & $40 / 110(36.4)$ & $<0.01$ \\
\hline \multicolumn{4}{|l|}{ Ischaemic events } \\
\hline Myocardial infarction* & $12 / 177(6.8)$ & $3 / 70(4.3)$ & 0.46 \\
\hline Ischaemic stroke* & $2 / 177(1.1)$ & $3 / 70(4.3)$ & 0.11 \\
\hline $\begin{array}{l}\text { Death or myocardial } \\
\text { infarction* or stroke* }\end{array}$ & 209/373 (56.0) & 45/110 (40.9) & $<0.01$ \\
\hline \multicolumn{4}{|l|}{ Bleeding events } \\
\hline $\begin{array}{l}\text { Combined GUSTO } \\
\text { bleedings* }\end{array}$ & 29/177 (16.4) & 10/70 (14.3) & 0.68 \\
\hline $\begin{array}{l}\text { GUSTO major } \\
\text { bleedings* }\end{array}$ & $4 / 177(2.3)$ & $3 / 70(4.3)$ & 0.39 \\
\hline \multicolumn{4}{|c|}{$\begin{array}{l}\text { Unadjusted death, ischaemic and bleeding events in the clopidogrel vs. } \\
\text { prasugrel/ticagrelor subgroups at } 12 \text { months. Data presented are } \\
\text { numbers of patients (percentages). *in surviving patients. }\end{array}$} \\
\hline
\end{tabular}

rate of mortality did not differ between prasugrel vs. clopidogrel treated patients (HR: 0.77, 95\% CI: 0.51-1.18, $\mathrm{p}=0.24$ ).

\section{Discussion}

This post hoc IABP-SHOCK II trial subgroup analysis represents the first large analysis of the safety and efficacy of the P2Y receptor inhibitor clopidogrel and the newer and more potent $\mathrm{P} 2 \mathrm{Y}_{12}$ receptor inhibitors prasugrel and ticagrelor in AMI patients complicated by cardiogenic shock. The key findings of our study at one-year follow-up are that: (a) the adjusted mortality risk did not differ in prasugrel/ticagrelor vs. clopidogrel treated patients, and (b) concerning safety aspects, there was no excess in bleeding complications for prasugrel and ticagrelor treated patients.

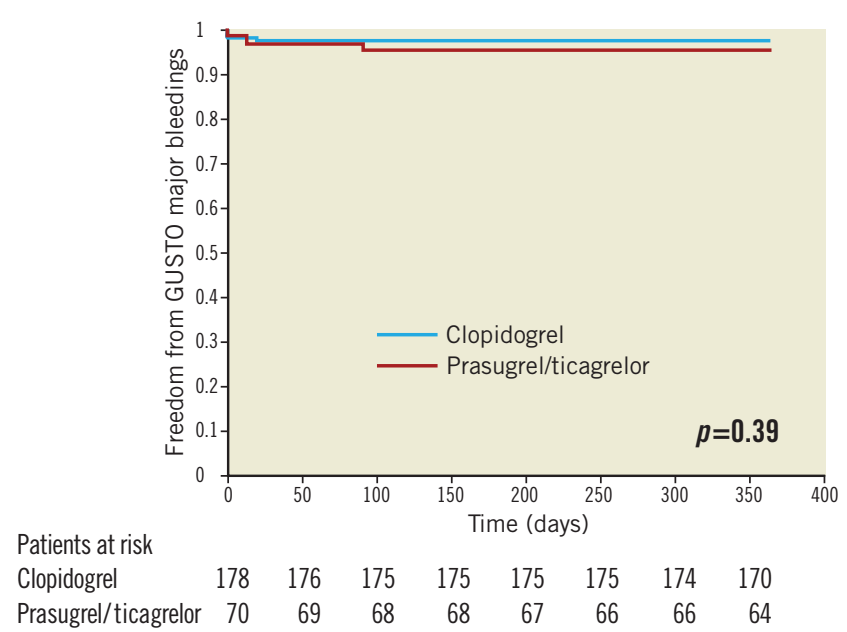

Figure 5. One-year freedom from GUSTO major bleedings. Kaplan-Meier curves of freedom from GUSTO major bleedings during one-year follow-up in patients treated with prasugrel or ticagrelor (red line) versus clopidogrel (blue line). 


\section{EFFICACY OF P2Y ${ }_{12}$ RECEPTOR INHIBITORS IN CARDIOGENIC SHOCK}

The lower unadjusted mortality rate in prasugrel/ticagrelor treated patients was mainly driven by significantly different baseline characteristics. Patients treated with clopidogrel were significantly older and sicker. Age was the strongest predictor of overall survival in this cohort of cardiogenic shock patients. This finding is in line with existing data ${ }^{16,17}$. After adjustment for confounders, no difference in mortality could be detected regarding $\mathrm{P} 2 \mathrm{Y}_{12}$ receptor inhibitor treatment.

The TRITON-TIMI 38 trial $^{3}$ and the PLATO trial ${ }^{4}$ excluded patients who suffered from cardiogenic shock complicating AMI. Thus, the data presented here are novel in this respect. The prespecified sub-analysis of STEMI patients ${ }^{18}$ from the TRITON-TIMI 38 trial $^{3}$ and the whole PLATO trial ${ }^{4}$ reported a survival benefit of patients treated with the more potent $\mathrm{P} 2 \mathrm{Y}_{12}$ receptor inhibitors prasugrel and ticagrelor compared to clopidogrel, respectively. The potent, faster and more reliable antiplatelet action of prasugrel and ticagrelor may benefit especially STEMI and shock patients, where drug absorption (accounting for both drugs) and in vivo bioactivation (accounting for prasugrel) is likely to be delayed and impaired. However, we did not detect a survival benefit with the use of these drugs. The reasons for this might be the lower patient number compared to the larger trials and the excessive overall mortality rate of cardiogenic shock patients.

One further important reason might be that acute mortality is not influenced by platelet inhibition by oral $\mathrm{P} 2 \mathrm{Y}_{12}$ receptor inhibitors and may be more influenced by haemodynamic status, inflammation, and end-organ failure. In the PRAGUE-7 trial - although not powered to show a difference in clinical outcome-even the more potent platelet inhibition with the GP IIb/IIIa inhibitor abciximab did not reduce mortality in patients with cardiogenic shock undergoing $\mathrm{PCI}^{19}$.

\section{SAFETY OF P2Y ${ }_{12}$ RECEPTOR INHIBITORS IN CARDIOGENIC SHOCK}

Cardiogenic shock patients are prone to suffer specifically from bleeding events due to their temporary need for resuscitation requiring repeated chest compression, insertion of a central venous catheter, arterial sheaths and the use of IABP requiring therapeutic anticoagulation, increasing the bleeding risk. Of note, we did not observe an excess of bleeding complications in the prasugrel/ticagrelor-treated subgroup of patients. This observation is in line with the sub-analysis of STEMI patients treated with prasugrel from TRITON-TIMI $38^{18}$, while it is in contrast to the results reported from the entire TRITONTIMI 38 study cohort ${ }^{3}$ and in contrast to ACS patients treated with ticagrelor within the PLATO trial ${ }^{4}$. Based on the above-mentioned observations, the use of prasugrel or ticagrelor might not be harmful in selected patients and could be endorsed for antiplatelet treatment of AMI patients complicated by cardiogenic shock.

Pharmacodynamic data on the antiplatelet action of clopidogrel, prasugrel and ticagrelor are limited in AMI patients complicated by cardiogenic shock. In this setting, several groups have shown a reduced antiplatelet efficacy of clopidogrel ${ }^{6,11}$ and of prasugrel ${ }^{11}$. Studies including patients after cardiac arrest who were treated with therapeutic hypothermia have indicated a reduced antiplatelet effect of clopidogre ${ }^{7-10}$ and of prasugrel and ticagrelor ${ }^{9}$ as well. Although we did not investigate pharmacodynamic effects of $\mathrm{P}_{2} \mathrm{Y}_{12}$ receptor inhibitors in the IABP-SHOCK II trial, the latter observations may explain - at least in part - both of our findings, as a possible uniform reduction of the antiplatelet action of all three drugs may lead to a weaker effect and may result in a lack of differences in the occurrence of ischaemic and bleeding events. Therefore, and according to the consensus document on invasive coronary treatment strategies for out-of-hospital cardiac arrest, the intravenously administered $\mathrm{P} 2 \mathrm{Y}_{12}$ receptor inhibitor cangrelor or GP IIb/IIIa inhibitors might be an alternative option in a cardiac shock condition, because their treatment efficacy does not require any prior active absorption or in vivo bioactivation ${ }^{20}$.

\section{Summary}

As a result of their high mortality risk and the difficulties of enrolling cardiogenic shock patients in randomised controlled trials, this cohort of patients is an understudied population with respect to $\mathrm{P} 2 \mathrm{Y}_{12}$ receptor inhibitor treatment. Results from prior trials cannot be extrapolated to this specific high-risk cohort of patients. Therefore, and in order to gain solid evidence on the safety and efficacy of potent platelet inhibition in shock patients, randomised studies are needed to determine the optimal $\mathrm{P} 2 \mathrm{Y}_{12}$ receptor inhibitor treatment regimen in patients with AMI complicated by cardiogenic shock and undergoing PCI. This IABP-SHOCK II trial subgroup analysis may provide the basis for such studies, as it is safe to administer potent $\mathrm{P} 2 \mathrm{Y}_{12}$ receptor inhibitors in the setting of cardiogenic shock.

\section{Limitations}

There are limitations of this study that merit being mentioned. First, this study is a post hoc analysis of a non-randomised study with all its known limitations. Although the differences in patient characteristics highlight a treatment bias between the two groups, a risk-adjusted survival analysis as well as an agespecific subgroup analysis were employed to mitigate these limitations. Furthermore, $\mathrm{P} 2 \mathrm{Y}_{12}$ receptor inhibitor treatment was not randomised in this subgroup analysis, and no information exists on $\mathrm{P} 2 \mathrm{Y}_{12}$ receptor inhibitor use after discharge. Although the number of patients treated with ticagrelor is low, this is the first study which analyses clinical outcome of ticagrelor-treated patients in cardiogenic shock. Due to the fact that ticagrelor does not require in vivo bioactivation, it might be speculated that its use is beneficial in a scenario of AMI complicated by cardiogenic shock. This warrants further investigation in future clinical trials. A potential confounder regarding acute antiplatelet effects of administered clopidogrel, prasugrel and ticagrelor could have been the broad use of GP IIb/IIIa inhibitors. Moreover, there was no routine platelet function monitoring of patients, so we could not investigate pharmacodynamic effects. Despite the fact that the number of patients in this sub-analysis is low, it provides an exceptional data 
set of the largest randomised controlled trial ever performed in patients with AMI complicated by cardiogenic shock and undergoing primary PCI in the vast majority of patients to analyse the safety of prasugrel and ticagrelor.

\section{Impact on daily practice}

This IABP-SHOCK II trial subgroup analysis shows that the use of potent $\mathrm{P} 2 \mathrm{Y}_{12}$ receptor inhibitors like prasugrel or ticagrelor is feasible and might not be harmful in selected patients with cardiogenic shock complicating AMI. However, the superiority in comparison to clopidogrel remains to be proven.

\section{Funding}

Institut für Herzinfarktforschung, Ludwigshafen; German Research Foundation; German Heart Research Foundation; German Cardiac Society Arbeitsgemeinschaft Leitende Kardiologische Krankenhausärzte; University of Leipzig - Heart Center. Unrestricted grants from Maquet Cardiopulmonary AG, Hirrlingen, Germany, and Teleflex Medical, Everett, MA, USA.

\section{Conflict of interest statement}

M. Orban reports receiving travel grants and speaker honoraria from Roche and travel grants from Abbott. M. Ferenc reports receiving speaker honoraria from Eli Lilly. G. Richardt reports receiving lecture fees from Maquet Cardiovascular. S. Schneider reports serving on the ethics committee of Landesärztekammer BadenWürttemberg, receiving payment for manuscript preparation from Biosense Webster, Grupo Ferrer, and Nycomed, and receiving money on behalf of the clinical research organisation at his institution from Abbott Vascular, AstraZeneca, Bayer Schering, Bayer Vital, Biotronik, Bristol-Myers Squibb, Boehringer Ingelheim, Cordis, Daiichi Sankyo, Diagenics, Enverdis, Eli Lilly, GlaxoSmithKline, Guidant, IKKF, Impulse Dynamics, Medtronic, Merck, Novartis, Roche Diagnostics, Sanofi Aventis, Schering-Plough, Siemens, St. Jude Medical, Takeda Pharma, Trommsdorff, and Vifor Pharma. $\mathrm{K}$. Werdan reports serving on the board of Biotest and Servier, receiving grant support on behalf of his institution from Biotest and Servier, and receiving lecture fees from Biotest, Brahms, Maquet Cardiovascular, and Servier. U. Zeymer reports serving on the board of Daiichi Sankyo and Eli Lilly and receiving consulting and lecture fees from Daiichi Sankyo, Eli Lilly, and The Medicines Company. H. Thiele reports receiving consulting fees from Eli Lilly, grant support on behalf of his institution from Eli Lilly and Terumo, and lecture fees from AstraZeneca, Boehringer Ingelheim, Daiichi Sankyo, Eli Lilly, The Medicines Company, and Terumo. J. Hausleiter has received speaker honoraria from Abbott Vascular. The other authors have no conflicts of interest to declare.

\section{References}

1. Task Force on the management of ST-segment elevation acute myocardial infarction of the European Society of Cardiology (ESC), Steg PG, James SK, Atar D, Badano LP, Blomstrom-Lundqvist C,
Borger MA, Di Mario C, Dickstein K, Ducrocq G, FernandezAviles F, Gershlick AH, Giannuzzi P, Halvorsen S, Huber K, Jüni P, Kastrati A, Knuuti J, Lenzen MJ, Mahaffey KW, Valgimigli M, van 't Hof A, Widimsky P, Zahger D. ESC Guidelines for the management of acute myocardial infarction in patients presenting with ST-segment elevation. Eur Heart J. 2012;33:2569-619.

2. Windecker S, Kolh P, Alfonso F, Collet JP, Cremer J, Falk V, Filippatos G, Hamm C, Head SJ, Jüni P, Kappetein AP, Kastrati A, Knuuti J, Landmesser U, Laufer G, Neumann FJ, Richter DJ, Schauerte P, Sousa Uva M, Stefanini GG, Taggart DP, Torracca L, Valgimigli M, Wijns W, Witkowski A. 2014 ESC/EACTS Guidelines on myocardial revascularization. EuroIntervention. 2015; 10:1024-94.

3. Wiviott SD, Braunwald E, McCabe $\mathrm{CH}$, Montalescot G, Ruzyllo W, Gottlieb S, Neumann FJ, Ardissino D, De Servi S, Murphy SA, Riesmeyer J, Weerakkody G, Gibson CM, Antman EM; TRITON-TIMI 38 Investigators. Prasugrel versus clopidogrel in patients with acute coronary syndromes. N Engl J Med. 2007;357: 2001-15.

4. Wallentin L, Becker RC, Budaj A, Cannon CP, Emanuelsson H, Held C, Horrow J, Husted S, James S, Katus H, Mahaffey KW, Scirica BM, Skene A, Steg PG, Storey RF, Harrington RA; PLATO Investigators, Freij A, Thorsen M. Ticagrelor versus clopidogrel in patients with acute coronary syndromes. $N$ Engl J Med. 2009;361:1045-57.

5. Thiele H, Zeymer U, Neumann FJ, Ferenc M, Olbrich HG, Hausleiter J, Richardt G, Hennersdorf M, Empen K, Fuernau G, Desch S, Eitel I, Hambrecht R, Fuhrmann J, Bohm M, Ebelt H, Schneider S, Schuler G, Werdan K; IABP-SHOCK II Trial Investigators. Intraaortic balloon support for myocardial infarction with cardiogenic shock. N Engl J Med. 2012;367:1287-96.

6. Osmancik P, Jirmar R, Hulikova K, Peroutka Z, Pompachova A, Motovska Z, Widimsky P. A comparison of the VASP index between patients with hemodynamically complicated and uncomplicated acute myocardial infarction. Catheter Cardiovasc Interv. 2010;75:158-66.

7. Bjelland TW, Hjertner O, Klepstad P, Kaisen K, Dale O, Haugen BO. Antiplatelet effect of clopidogrel is reduced in patients treated with therapeutic hypothermia after cardiac arrest. Resuscitation. 2010;81:1627-31.

8. Souckova L, Opatrilova R, Suk P, Cundrle I Jr, Pavlik M, Zvonicek V, Hlinomaz O, Sramek V. Impaired bioavailability and antiplatelet effect of high-dose clopidogrel in patients after cardiopulmonary resuscitation (CPR). Eur J Clin Pharmacol. 2013;69: 309-17.

9. Ibrahim K, Christoph M, Schmeinck S, Schmieder K, Steiding K, Schoener L, Pfluecke C, Quick S, Mues C, Jellinghaus S, Wunderlich C, Strasser RH, Kolschmann S. High rates of prasugrel and ticagrelor non-responder in patients treated with therapeutic hypothermia after cardiac arrest. Resuscitation. 2014;85:649-56.

10. Steblovnik K, Blinc A, Bozic-Mijovski M, Kranjec I, Melkic E, Noc M. Platelet reactivity in comatose survivors of cardiac arrest undergoing percutaneous coronary intervention and hypothermia. EuroIntervention. 2015;10:1418-24. 
11. Orban M, Mayer K, Morath T, Bernlochner I, Hadamitzky M, Braun S, Schulz S, Hoppmann P, Hausleiter J, Tiroch K, Mehilli J, Schunkert H, Massberg S, Laugwitz KL, Sibbing D, Kastrati A. Prasugrel vs clopidogrel in cardiogenic shock patients undergoing primary PCI for acute myocardial infarction. Results of the ISARSHOCK registry. Thromb Haemost. 2014;112:1190-7.

12. Thiele H, Schuler G, Neumann FJ, Hausleiter J, Olbrich HG, Schwarz B, Hennersdorf M, Empen K, Fuernau G, Desch S, de Waha S, Eitel I, Hambrecht R, Bohm M, Kurowski V, Lauer B, Minden HH, Figulla HR, Braun-Dullaeus RC, Strasser RH, Rochor K, Maier SK, Mollmann H, Schneider S, Ebelt H, Werdan K, Zeymer U. Intraaortic balloon counterpulsation in acute myocardial infarction complicated by cardiogenic shock: design and rationale of the Intraaortic Balloon Pump in Cardiogenic Shock II (IABPSHOCK II) trial. Am Heart J. 2012;163:938-45.

13. Van de Werf F, Bax J, Betriu A, Blomstrom-Lundqvist C, Crea F, Falk V, Filippatos G, Fox K, Huber K, Kastrati A, Rosengren A, Steg PG, Tubaro M, Verheugt F, Weidinger F, Weis M; ESC Committee for Practice Guidelines (CPG). Management of acute myocardial infarction in patients presenting with persistent ST-segment elevation: the Task Force on the Management of ST-Segment Elevation Acute Myocardial Infarction of the European Society of Cardiology. Eur Heart J. 2008;29:2909-45.

14. Werdan K, Russ M, Buerke M, Delle-Karth G, Geppert A, Schondube FA; German Cardiac Society; German Society of Intensive Care and Emergency Medicine; German Society for Thoracic and Cardiovascular Surgery; (Austrian Society of Internal and General Intensive Care Medicine; German Interdisciplinary Association of Intensive Care and Emergency Medicine; Austrian Society of Cardiology; German Society of Anaesthesiology and Intensive Care Medicine; German Society of Preventive Medicine and Rehabilitation. Cardiogenic shock due to myocardial infarction: diagnosis, monitoring and treatment: a German-Austrian S3 Guideline. Dtsch Arztebl Int. 2012;109:343-51.

15. [No authors listed]. An international randomized trial comparing four thrombolytic strategies for acute myocardial infarction. The GUSTO investigators. N Engl J Med. 1993;329:673-82.

16. Hasdai D, Holmes DR Jr, Califf RM, Thompson TD, Hochman JS, Pfisterer M, Topol EJ. Cardiogenic shock complicating acute myocardial infarction: predictors of death. GUSTO Investigators. Global Utilization of Streptokinase and TissuePlasminogen Activator for Occluded Coronary Arteries. Am Heart J. 1999;138:21-31.
17. Zeymer U, Vogt A, Zahn R, Weber MA, Tebbe U, Gottwik M, Bonzel T, Senges J, Neuhaus KL; Arbeitsgemeinschaft Leitende Kardiologische Krankenhausärzte (ALKK). Predictors of in-hospital mortality in 1333 patients with acute myocardial infarction complicated by cardiogenic shock treated with primary percutaneous coronary intervention (PCI); Results of the primary PCI registry of the Arbeitsgemeinschaft Leitende Kardiologische Krankenhausarzte (ALKK). Eur Heart J. 2004;25:322-8.

18. Montalescot G, Wiviott SD, Braunwald E, Murphy SA, Gibson CM, McCabe CH, Antman EM; TRITON-TIMI 38 investigators. Prasugrel compared with clopidogrel in patients undergoing percutaneous coronary intervention for ST-elevation myocardial infarction (TRITON-TIMI 38): double-blind, randomised controlled trial. Lancet. 2009;373:723-31.

19. Tousek P, Rokyta R, Tesarova J, Pudil R, Belohlavek J, Stasek J, Rohac F, Widimsky P. Routine upfront abciximab versus standard periprocedural therapy in patients undergoing primary percutaneous coronary intervention for cardiogenic shock: The PRAGUE-7 Study. An open randomized multicentre study. Acute Card Care. 2011;13:116-22.

20. Noc M, Fajadet J, Lassen JF, Kala P, MacCarthy P, Olivecrona GK, Windecker S, Spaulding C; European Association for Percutaneous Cardiovascular Interventions (EAPCI); Stent for Life (SFL) Group. Invasive coronary treatment strategies for outof-hospital cardiac arrest: a consensus statement from the European association for percutaneous cardiovascular interventions (EAPCI)/stent for life (SFL) groups. EuroIntervention. 2014;10:31-7.

\section{Supplementary data}

Appendix. Patients with available information on $\mathrm{P}_{2} \mathrm{Y}_{12}$ receptor inhibitor treatment. Study population.

Appendix Table 1. Baseline characteristics of the patients.

Appendix Table 2. Clinical presentation of both subgroups prior to randomisation during the primary IABP-SHOCK II trial.

Appendix Table 3. Univariate and multivariate Cox model of 12-month mortality.

Appendix Table 4. Clinical outcome at one year.

Appendix Figure 1. Study flow chart.

Appendix Figure 2. Unadjusted one-year survival.

Appendix Figure 3. One-year freedom from all GUSTO bleedings. Appendix Figure 4. One-year freedom from GUSTO major bleedings. 